www.jmscr.igmpublication.org

Impact Factor 5.84

Index Copernicus Value: 83.27

ISSN (e)-2347-176x ISSN (p) 2455-0450

crossref DOI: _https://dx.doi.org/10.18535/jmscr/v5i6.116

Journal Of Medical Science And Clinical Research

IGM Publication

An official Publication of IGM Publication

\title{
Role of Computed Tomography in Diagnosis of Bronchogenic Carcinoma with Histopathological Correlation
}

\author{
Authors \\ Dr Adaikkalavan C1, Dr Sajitha $K^{2}$, Dr Josey Verghese ${ }^{3}$, Dr Amilu Elsa Varghese \\ ${ }^{1}$ Formerly Junior Residenst in Radiodiagnosis, Govt. T. D. Medical College, Alappuzha \\ ${ }^{2}$ Associate Professor of Radiodiagnosis, Medical College, Kottayam \\ ${ }^{3}$ Professor of Radiodiagnosis, Govt. T. D. Medical College, Alappuzha \\ ${ }^{4}$ Formerly Junior Resident in Radiodiagnosis, Govt. T. D. Medical College, Alappuzha \\ Corresponding Author

\section{Dr Sajitha K}

Department of Radiodiagnosis, Govt. Medical College, Kottayam, Kerala, India

PH: 9447596346, FAX: Email:drsajithak@gmail.com

\section{ABSTRACT}

Background: In India, lung cancer is the commonest and most lethal cancer among males accounting for $10.9 \%$ of all cancer cases and $13 \%$ of cancer related mortality. Computed tomography is the modality of choice for evaluating bronchogenic carcinoma. CT has a role in identifying the lung nodules and characterizing them as benign or malignant. CT guided FNAC of lung lesions has rapidly emerged as less invasive, cheap, rapid \& fairly accurate diagnostic aid in lung lesions. The present study is aimed at evaluating the imaging characteristics of pulmonary mass lesions by CT along with CT guided FNAC/ bronchoscopy guided biopsy of the lesions for histopathological correlation thereby aiding patients in correct diagnosis and thereby aiding patients with appropriate treatment also.

Materials and methods: Data was collected from 53 patients with clinically or radiologically suspected pulmonary mass lesion referred for CT scan of thorax to Department of Radiodiagnosis. Contrast study is done, lung lesions were characterized and CT guided FNAC done in our Department using 22G Spinal needle under local anaesthesia. Collected data were analysed based on the variables of the lung lesion seen in CT images followed by CT diagnosis and correlation made with histopathology report.

Conclusion: CT guided FNAC is a simple, less invasive and effective method to obtain Histopathology thereby prompting appropriate management. CT is a highly sensitive test (95.83\%) and also has a high positive predictive value (97.87\%) to diagnose Bronchogenic carcinoma in a sample suitably selected and referred for malignancy. Diagnostic work up by CT with guided FNAC may help the patient in correct diagnosis and thereby prompting appropriate management.

Keywords: Bronchogenic carcinoma, CT guided FNAC, Bronchoscopy guided biopsy

\section{INTRODUCTION}

Bronchogenic carcinoma remains the most common cause of cancer death worldwide. The incidence of the disease has accelerated over the past century, closely in step with tobacco smoking. The most commonly diagnosed cancers worldwide are lung (1.61 million, $12.7 \%$ of the total), breast (1.38 million,10.9\%) and colorectal cancers (1.23 million,9.7\%). The most common cause of cancer death is lung cancer (1.38 million, $18.2 \%$ of the total). Based on the biology, therapy 
and prognosis, lung cancer is broadly divided into two classes - non-small cell lung cancer (NSCLC) and small cell lung cancer (SCLC). Squamous cell carcinoma, adenocarcinoma and large cell carcinoma are classified as NSCLC and account for $75 \%$ to $80 \%$ of all lung cancer cases. For early stage NSCLC, surgery is almost curative.. The challenge is, thus, to identify the disease at its earliest stage when it is still amenable to treatment. ${ }^{1}$

\section{Classification}

A practical classification based on the treatment options for bronchogenic carcinoma divides the histologic types into non-small cell and small cell carcinomas of which the latter responds to chemotherapy and radiotherapy.

\section{Non-small cell Lung cancer (NSCLC):}

\section{Adenocarcinoma : Adenocarcinoma is the} most common cell type accounting for 30-35\% of all lung cancers and is the most common cell type in nonsmokers. Early metastasis is more common than with squamous cell carcinoma, particularly to the central nervous system and adrenal glands. Seventy-five percent originate in the lung periphery, presenting as a solitary pulmonary nodule or mass. In a few cases, adenocarcinoma originates within large airways. It is most common in the upper lobes. It is often associated with fibrosis; it may arise in relation to preexisting lung fibrosis (i.e. a scar carcinoma) or may result in a desmoplastic reaction in surrounding lung. Adenocarcinomas often appear ill defined on chest radiographs because of their irregular margin. On high-resolution CT, lesions appear round or lobulated. They frequently have an irregular and spiculated margin because of associated lung fibrosis. When occurring in a subpleural location, this may result in thin linear extensions to the pleural surface (i.e., a pleural tail). Air bronchograms may be visible within the nodule using CT. Most of the lesions are asymptomatic due to their peripheral location. It may be associated with concomitant lung disease, such as focal and diffuse fibrosis.

The new classification now divides adenocarc- inoma into the following categories:

Pre-malignant lesions: This includes atypical adenomatous hyperplasia and adenocarcinoma in situ. These lesions are $3 \mathrm{~cm}$ or less in diameter and histology manifests pure lepidic growth with no solid components. These will appear as pure GGO on CT.

Malignant lesions, divided into the following:

Minimally invasive adenocarcinoma, with predominantly (in distinction to pure) lepidic growth, $3 \mathrm{~cm}$ or less, and with invasive components of no more than $5 \mathrm{~mm}$. These are generally sub-solid nodules on CT.

Invasive adenocarcinomas, subclassified as predominantly lepidic, acinar, papillary, micropapillary and solid types.

Invasive mucinous adenocarcinoma, an entity formerly described as mucinous bronchioalveolar carcinoma and considered as a separate group from the non-mucinous types above.

Prognosis of patients with adenocarcinoma in situ or minimally invasive adenocarcinoma (characterised on CT as pure ground-glass lesions) is excellent; these patients should have almost $100 \%$ disease-free survival. Invasive adenocarcinoma has a variable outlook, and to some extent this depends on the histological subtype.

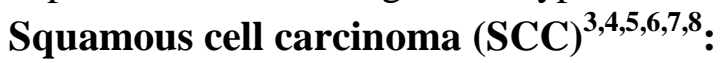

Squamous cell carcinoma contributes to approximately $30 \%$ of all lung cancers. It is associated with the best prognosis. Although it generally shows rapid local growth but distant metastases occur at a later phase. It has a strong association with smoking. At the time of detection, most tumors are between 3 and $5 \mathrm{cms}$ and centrally located, resulting in post obstructive atelectasis or pneumonia. Squamous cell carcinoma is associated with the presence of amorphous, punctate calcification within the lesion and the presence of cavitation. Cavity walls are usually thick, nodular and irregular, ranging in size from 0.5 to $3 \mathrm{~cm}$. Rarely, extensive necrosis may result in a thin-walled cavity. Patients also present with signs and symptoms due to invasion of adjacent central structures, such as involvement of the recurrent laryngeal nerve.. 
Pancoast syndrome comprises of involvement of the brachial plexus and cervical sympathetic nerves associated with severe pain in the shoulder region radiating toward the axilla and scapula; atrophy of hand and arm muscles; Horner's syndrome is a constellation of signs produced because of interruption of the sympathetic innervation; and compression of blood vessels with edema. Pancoast tumor can occur with any histology but are more commonly seen with both squamous cell carcinoma and adenocarcinoma.

The term "superior sulcus" tumor refers to its location in the superior pulmonary sulcus at the lung apex, from which it can cause local invasion of the chest wall and brachial plexus.

Large cell carcinoma : The term large cell carcinoma is used to describe tumors that do not show squamous or adenomatous differentiation or have typical features of small cell carcinoma. Large cell carcinoma accounts for $10 \%$ of lung cancers. It tends to present as a large peripheral mass; more than $60 \%$ are larger than $4 \mathrm{~cm}$ at presentation. It is similar to adenocarcinoma in its radiologic characteristics (except for its large size), histologic ultrastructure, and survival statistics. As with adenocarcinoma, it tends to metastasize early and has a poor prognosis. It is strongly associated with smoking.

Small cell lung cancer (SCLC) ${ }^{\mathbf{3 , 4 , 9}}$ : Small cell lung cancer, formerly known as "oat cell carcinoma" for the small, round shape of the cancer cells, is an aggressive tumor which usually presents with generalized symptoms and distant metastases. Although these tumors respond initially to chemotherapy, later on most patients develop drug resistance. Small Cell Lung Cancer is responsible for approximately $20 \%$ all lung cancer cases. Small cell carcinoma tends to occur in the main or lobar bronchi and is associated with extensive peribronchial invasion and a large hilar or parahilar mass. Endobronchial tumor masses are less common than with squamous cell carcinoma, but the large tumor mass frequently compresses bronchi. Atelectasis may be associated. This tumor is commonly associated with marked mediastinal lymph node enlargement. It is a common cause of superior vena cava (SVC) syndrome. Presentation as a lung nodule is very uncommon, accounting for less than $5 \%$ of cases. Over $90 \%$ of cases are stage IV at diagnosis.

Multiple primary carcinomas ${ }^{3,4}$ : Synchronous lesions are defined as the presence of two tumors at the same time or closely following initial diagnosis. The incidence of synchronous multiple primary tumors represents less than $3.5 \%$ of all lung cancers. This number may even be higher depending on the cell type and how carefully further primary tumors are looked for and the rigidity of the criteria used to define the tumors as primary lesions. Difference in cell type is an accepted criterion. For tumors of the same histologic type they must be separated by noncancerous lung tissue.

Metachronous lesions are defined as the second cancers appearing after a time interval, usually12 months or more. The peak incidence is between the third and eighth postoperative years. These lesions constitute at least two thirds of multiple pulmonary neoplasms. Ten percent to thirty two percent of patients surviving resection for lung cancer may develop a second primary tumor. The reported incidence has increased probably because second primary lung cancer can be distinguished from recurrence and satellite disease due to better investigations. These lesions are regarded as multiple primary lesions only if they show unique histologic features. Adenocarcinoma has replaced squamous cell carcinoma as the most common histologic type in multiple carcinoma.

Imaging features of bronhogenic carcinoma ${ }^{10}$ : Peripheral tumours: Approximately $40 \%$ of bronchial carcinomas arise beyond the segmental bronchi, and in $30 \%$ a peripheral mass is the sole radiographic finding.

Tumour Shape and Margins: Tumours at the lung apex (Pancoast's tumours, superior sulcus tumours) may resemble apical pleural thickening; however, the majority of peripheral lung cancers are approximately spherical or oval in shape. 
Lobulation, a sign that indicates uneven growth rates in different parts of the tumour, is common. Occasionally, a dumb-bell shape is encountered or two nodules are seen next to one another. The term 'corona radiata' is used to describe numerous fine strands radiating into the lung from a central mass, sometimes with transradiant lung parenchyma between these strands. While not specific, this sign is a highly suggestive of bronchial carcinoma. Absolutely spherical, sharply defined, smooth-edged nodules due to carcinoma of the lung are rare. A peripheral line shadow or 'tail' may be seen between a peripherally located mass lesion and the pleura, a phenomenon that occurs in both benign and malignant lesions. When associated with carcinoma of the lung, the 'tail' probably represents either plate-like atelectasis secondary to bronchial obstruction beyond the mass or septal oedema due to lymphatic obstruction. Although the edges of a tumour are frequently well defined, some peripheral cancers, notably some types of adenocarcinoma, have ill-defined edges similar to pneumonia.

Cavitation: Cavitation may be identified in tumours of any size and is best demonstrated by CT. Squamous cell carcinoma is the most likely cell type to show cavitation. The walls of the cavity are of irregular thickness and may contain tumour nodules, but sometimes the wall has smooth inner and outer margins. The cavity wall is usually $8-\mathrm{mm}$ thick or greater. Fluid levels are common.

Calcification: Calcification within bronchogenic carcinomas is rarely seen on chest radiography but is identified on CT in $6-10 \%$ of cases. Some foci of calcification represent preexisting calcified granulomatous disease engulfed by tumour. However, amorphous or cloud-like calcification consistent with dystrophic tumour calcification is still seen in a small proportion of cases $(<10 \%)$. Most calcified tumours are large with a diameter of $5 \mathrm{~cm}$ or more, but calcification can also be seen in small peripheral tumours.

Other Findings: Air bronchograms and bubblelike lucencies or pseudocavitation may be seen within lung cancers, in particular with adenocarcinoma. Occasionally, dilated mucus filled bronchi (bronchocele, mucocele, mucoid impaction) are seen distal to a carcinoma obstructing a segmental or subsegmental bronchus. Ground-glass attenu-ation may be seen as a component of nodules and is associated with a greater risk of malignancy than that of purely solid nodules. It is more commonly associated with adenocarcinoma, which may present as apurely ground-glass opacity.

Central tumours: The cardinal imaging signs of a central tumour are collapse/consolidation of the lung beyond the tumour and the presence of hilar enlargement, signs that may be seen in isolation or in conjunction with one another.

Collapse/Consolidation in Association with Central Tumours: Obstruction of a major bronchus often leads to a combination of atelectasis and retention of secretions with consequent pulmonary opacity, but collateral air drift may partially or completely prevent these post obstructive changes. Secondary infection may occur beyond the obstruction. The following features suggest that pneumonia is secondary to an obstructing neoplasm:

1. The shape of the collapsed or consolidated lobe may be altered because of the bulk of the underlying tumour. In cases with lobar collapse due to a central tumour mass, the fissure in the region of the mass is unable to move in the usual manner and, therefore, the fissure may show a bulge (the Golden S sign if involving the right upper lobe).The description now seems to have become extended to include the CT equivalent appearance of a hilar mass and collapsed distal lobe and also to describe the phenomenon in other lobes.

2. The presence of pneumonia in an at-risk patient, confined to one lobe (or more lobes if there is a common bronchus) that persists unchanged for longer than 2-3 weeks, or a pneumonia that recurs in the same lobe, particularly if the lobe shows loss of volume and no air bronchograms. 
Simple pneumonia often clears or spreads to other segments within a few weeks. In practice, complete resolution of pneumonia virtually excludes an obstructing neoplasm as a cause of infection. Although consolidation may improve partially on appropriate antibiotic therapy, it almost never resolves completely if secondary to an underlying carcinoma. Occasionally, the opacified lobe may appear larger than normal because of the build-up of infected secretions beyond the obstructing carcinoma, an appearance that has been labelled the 'drowned lobe'.

3. A visible mass with irregular stenosis of a mainstem or lobar bronchus. Careful analysis of CT images may demonstrate the presence of an obstructing tumour when there is obstructive atelectasis.

4. Simple pneumonia rarely causes radiographically visible hilar adenopathy, though enlarged central nodes may be seen on CT or MRI. Lung abscess can occasionally be confused with bronchial carcinoma because it may result in hilar or mediastinal adenopathy.

5. Mucus-filled dilated bronchi may be visible within collapsed lobes on a CT examination as branching, tubular lowdensity structures, and when seen should prompt a search for a centrally obstructing tumour.

Hilar Enlargement: Hilar enlargement is a common presenting feature in patients with bronchial carcinoma. It may reflect a proximal tumour, lymphadenopathy, consolidated lung, or a combination of these phenomena. A mass superimposed on the hilum may lead to increased density of the hilum, owing to summation of the opacity of the mass and that of the normal hilar shadows. This sign may be the only indication of lung cancer on a frontal chest radiograph; when suspected, it is essential to inspect the lateral radiograph with care.

Mediastinal Invasion: Plain radiograph evidence of mediastinal invasion relies on demonstrating phrenic nerve paralysis. Caution is needed, however, before deciding that a high hemidiaphragm is caused by phrenic nerve invasion, because lobar collapse can also lead to elevation of a hemidiaphragm, a subpulmonary effusion may mimic it,and diaphragmatic eventration is common. The major CT signs of mediastinal invasion include the demonstration of visible tumour deep within the mediastinal fat, particularly if tumour surrounds the mediastinal vessels, oesophagus, or proximal mainstem bronchi. Associated pneumonia or atelectasis may make it very difficult to determine whether or not mediastinal contact is present. Even clear-cut contact with the mediastinum is not enough for the diagnosis of invasion, and the apparent interdigitation of tumour with mediastinal fat can be a misleading sign on both CT. Glazer et al. showed that the presence of (A) less than $3 \mathrm{~cm}$ of contact with the mediastinum,(B) less than $90^{\circ}$ of circumferential contact with the aorta, or (C) a visible mediastinal fat plane between the mass and any vital mediastinal structures indicated a very high likelihood of technical resectability, even if the tumour had crossed into the mediastinum, and that most tumours in their series conforming to this description had no mediastinal invasion at surgery. When the question is turned round to enquire as to the criteria for unresectability, however, the answer is less certain. Tumours that obliterate fat planes or show greater contact than that described above are not necessarily unresectable, though the greater the degree of invasion and the extent of contact, the more

Chest Wall Invasion: The presence of chest wall invasion alone does not preclude surgical resection, though it does adversely affect prognosis. The necessarily more extensive surgery is associated with increased morbidity and mortality and it therefore helps the surgeon to know the extent of any chest wall invasion preoperatively. The diagnosis of chest wall involvement adjacent to a tumour is unreliable on $\mathrm{CT}$, unless there is clear- cut bone destruction or a large soft-tissue mass. Local chest wall pain 
remains the single most specific indicator of whether or not the tumour has spread to the parietal pleura or chest wall. Contact with the pleura on CT examination, even if the pleura is thickened, does not necessarily indicate invasion, though the greater the degree of contact and the greater the pleural thickening, the more likely it is that the parietal pleura has been invaded, particularly if the extrapleural fat plane is obliterated. A definite extrapleural mass that is not explicable by previous chest trauma is likely to be the result of invasion by tumour, but even this sign may be misleading since soft- tissue swelling may be due to inflammation and fibrosis rather than neoplasm. Conversely, a clear extrapleural fat plane adjacent to the mass may be helpful, but again not definitive, in excluding chest wall invasion.

\section{Lymph Node Assessment: Lymph node} assessment on CT and MRI is limited to size, shape and location, with size being the major criterion used to predict metastatic involvement. Normal mediastinal lymph node size on CT or MRI varies according to the location of the nodes within the mediastinum, but a simple and reasonably accurate rule is that nodes with a shortaxis diameter of less than $10 \mathrm{~mm}$ fall within the 95th percentile and nodes above this size should, therefore, be considered enlarged.

Pleural Involvement: Pleural involvement may occur as a result of direct spread, lymphatic involvement, or tumour emboli. On occasion, adenocarcinoma takes the form of a sheet of lobular pleural thickening indistinguishable from malignant mesothelioma. A pleural effusion in association with a primary lung cancer designates the tumour as being M1a. The exception is the few patients who have clinical evidence of another cause for the effusion (e.g. heart failure) and in whom cytology examinations of multiple pleural fluid samples are negative for tumour cells, in which case the effusion can be disregarded as a staging criterion.

Extra Thoracic involvement: Lung cancer is commonly associated with widespread haematogenous dissemination at the time of presentation. Sites of spread include the adrenal glands, bones, brain, liver and more distant lymph nodes. Detection of metastatic disease precludes surgical resection of the primary tumour. In most centres chest CT is extended to include the liver and adrenals (with appropriate timing for portal venous enhancement). Further imaging is usually only undertaken if there are clinical features suggesting metastatic disease.

Tissue sampling and arriving at final diagnosis $^{11}$

Tissue sampling of nodules which are both accessible and of a size, shape and morphology to suggest the possibility of malignancy, may be undertaken transbronchially, surgically or percutanously with radiological guidance. Central lesions may be amenable to bronchoscopic biopsy but even perihilar nodules, in the absence of an endoluminal component,remain very challenging for bronchoscopic diagnosis. Bronchoscopic biopsy of peripheral lung cancer using conventional techniques is also highly problematic.

Percutaneous fine needle or cutting needle biopsy has been demonstrated as an accurate technique for the identification of malignancy, but not all nodules are suitable for this approach. When a final diagnosis of lung cancer is eventually established, a previous aspiration biopsy has a 90\% likelihood of providing confirmation of a malignant diagnosis. There is a very low falsepositive rate but there is a recognised and troubling false-negative rate. Transthoracic needle biopsy remains an essential tool in the management part of indeterminate nodules. Multidisciplinary team discussion of the relative merits of a follow-up strategy, further imaging, percutaneous or bronchoscopic diagnostic procedures or surgical resection for an individual lung nodule remains the ideal management step in this common problem.

FNAC has proven its role in diagnosis of infections \& other diffuse benign processes although the main indication remains the diagnosis of localised intra- thoracic lesions suspected of being malignant. 


\section{MATERIALS AND METHODS}

The Study was designed as Diagnostic test evaluation and conducted at CT Scan centre, Department of Radiodiagnosis Alappuzha

Inclusion criteria: 1.Patients with clinically or radiologically suspected pulmonary mass lesion who came for CT thorax to Department of radiodiagnosis for evaluation and upon $\mathrm{CT}$ imaging were found to have pulmonary mass lesion which is defined as - "A focal pulmonary lesion which is greater than $3 \mathrm{~cm}$ in diameter in CT Thorax". 2.Patients in whom histopathological correlation is available.

Exclusion criteria: 1. Patients with pulmonary mass lesion in whom Histopathology is already proved. 2. Patients with history of contrast allergy. 3. Patients with deranged renal function test

Sample size: The number of patients satisfying the inclusion criteria within the duration of the study were fifty three.

All scans were done using Siemens Somatom Spirit dual slice spiral CT scan with $130 \mathrm{KVp}$ and 40-60mAs with $5 \mathrm{~mm}$ section thickness, retro reconstruction of $1 \mathrm{~mm}$ section thickness and reformation. Contrast study is done using $50 \mathrm{ml}$ of $300 \mathrm{mgI} / \mathrm{ml}$ non-ionic iodinated contrast, injected manually after $0.1 \mathrm{ml}$ intradermal test dose and $1 \mathrm{ml}$ intravenous test dose which is the routine followed in our CT department for all contrast studies. Sections were taken from the level of lung apices to the diaphragm routinely including the liver and adrenals.

Lung lesions were characterised based on the following variables- site, size, margins, enhancement pattern, presence of calcification, cavitation, involvement of the hila, involvement of pleura, involvement of chest wall, involvement of mediastinum, involvement of bone, involvement of liver and involvement of adrenals. CT findings were compared with histopathological examination of the specimen obtained by anyone of the following methods: CT guided FNAC done in our Department using 22G Spinal needle under local anaesthesia with aseptic precautions in accessible cases after obtaining proper informed consent. Arteriovenous malformations, pulmonary artery hypertension, bleeding disorder were ruled out before doing the procedure. Patients were observed for a period of 1 hour after FNAC to keep track of any complication. Repeat CT was performed in cases with suspected complication like pneumothorax post procedure.

Bronchoscopic Biopsy of the lesion/open surgical removal of the lesion which is usually done for the patient as a part of his/her management by respiratory medicine Department or cardiothoracic Department of depending upon the site of the lesion.

\section{Statistical analysis}

Collected data were analysed based on the variables of the lung lesion seen in CT images as already mentioned followed by CT diagnosis and staging and correlation made with histopathology report.

As the study design is diagnostic test evluation, collected data were analysed by calculating sensitivity, specificity, positive predictive value, negative predictive value using MedCalc software.

\section{RESULT}

Data collected from 53 patients suspected to have bronchogenic carcinoma in whom CT Thorax and tissue sampling was done by either $\mathrm{CT}$ guided FNAC or by Brochoscopic biopsy of the lesion/open surgical removal of the lesion. Out of this 53 patients, 47 were diagnosed to have Bronchogenic carcinoma by CT based on imaging findings and 48 patients were Histopathologically proven to have Bronchogenic carcinoma.

Table.1.Distribution of subjects based on size of the mass lesion

\begin{tabular}{|l|c|c|}
\hline Size & Frequency & Percent \\
\hline $3-7 \mathrm{cms}$ & 33 & 62.3 \\
\hline $77 \mathrm{cms}$ & 20 & 37.7 \\
\hline Total & 53 & 100.0 \\
\hline
\end{tabular}


Table.2. Comparison between margins and histopathology

\begin{tabular}{|l|c|c|c|c|c|c|}
\hline \multirow{2}{*}{ Margins } & \multicolumn{5}{|c|}{ Histopathology } & Total \\
\cline { 2 - 6 } & $\begin{array}{c}\text { Adeno } \\
\text { carcinoma }\end{array}$ & $\begin{array}{c}\text { Squamou } \\
\text { s cell }\end{array}$ & $\begin{array}{c}\text { small cell } \\
\text { carcinoma }\end{array}$ & $\begin{array}{c}\text { large cell } \\
\text { carcinoma }\end{array}$ & $\begin{array}{c}\text { Other } \\
\text { s }\end{array}$ & \\
\hline Spiculated & 10 & 15 & 6 & 1 & 2 & 34 \\
\hline Lobulated & 3 & 7 & 2 & 0 & 2 & 14 \\
\hline Smooth & 0 & 0 & 0 & 0 & 1 & 1 \\
\hline Irregular & 1 & 2 & 1 & 0 & 0 & 4 \\
\hline Total & 14 & 24 & 9 & 1 & 5 & 53 \\
\hline
\end{tabular}

Table.3 Distribution of subjects based on cavitation of the mass lesion in CT

\begin{tabular}{|l|c|c|}
\hline Cavitation & Frequency & Percent \\
\hline Present & 10 & 18.9 \\
\hline Absent & 43 & 81.1 \\
\hline Total & 53 & 100.0 \\
\hline
\end{tabular}

Table.4.Distribution of subjects based on Contrast enhancement of the mass lesion in CT

\begin{tabular}{|l|c|c|}
\hline Contrast enhancement & Frequency & Percent \\
\hline mild $(<20 \mathrm{HU})$ & 12 & 22.6 \\
\hline moderate $(20-40 \mathrm{HU})$ & 40 & 75.5 \\
\hline marked $(>40 \mathrm{HU})$ & 1 & 1.9 \\
\hline no enhancement & 0 & 0 \\
\hline Total & 53 & 100.0 \\
\hline
\end{tabular}

Table.5 Distribution of subjects based on CT diagnosis of Bronchogenic carcinoma

\begin{tabular}{|l|c|c|}
\hline CT diagnosis & Frequency & Percent \\
\hline Present & 47 & 88.7 \\
\hline Absent & 6 & 11.3 \\
\hline Total & 53 & 100.0 \\
\hline
\end{tabular}

Table.6 Distribution of subjects based on Histopatholgy of CT lesion

\begin{tabular}{|l|c|c|}
\hline Histopathology & Frequency & Percent \\
\hline Adeno carcinoma & 14 & 26.4 \\
\hline Squamous cell carcinoma & 24 & 45.3 \\
\hline Small cell carcinoma & 9 & 17.0 \\
\hline Large cell carcinoma & 1 & 1.9 \\
\hline Others & 5 & 9.4 \\
\hline Total & 53 & 100.0 \\
\hline
\end{tabular}

Method of specimen collection was by CT guided FNAC in 44 cases, Bronchoscopic biopsy in 8 cases and open Thoracotomy with wedge resection in 1 case.

The case in which wedge resection was done, HPR came as Bronchopulmonary sequestration which was diagnosed as necrotic malignant mass by CT.

Diagnostic test evaluation : $\mathrm{CT}$ diagnosis of the mass lesion to be Bronchogenic carcinoma was made when the lesion had the following features either alone or in combinations depending upon the case : spiculated margins, irregular thick walled cavitation, pseudocavitation, abrupt bronchus cut off with distal collapse consolidation and a differentially enhancing lesion, associated hilar/ mediastinal/ supraclavicular lypmhadenopathy, chest wall/ mediastinal / pleural invasion by the mass lesion with or without pleural effusion/pleural nodules,adjacent interstitial septal thickening (Lymphangitis carcinomatosis), separate nodules in ipsilateral or contralateral lung,distal metastatic lesion in adrenals/liver/bone.

Table.7 Sensitivity, Specificity, Positive predictive value, Negative predictive value of CT to diagnose Bronchogenic carcinoma

\begin{tabular}{|c|c|c|c|c|}
\hline \multirow{2}{*}{\multicolumn{2}{|c|}{, }} & \multicolumn{2}{|c|}{$\begin{array}{c}\text { Histopathology for } \\
\text { Bronchogenic carcinoma }\end{array}$} & \multirow[t]{2}{*}{ Total } \\
\hline & & Yes & No & \\
\hline \multirow{3}{*}{$\begin{array}{l}\text { CT for Y Y } \\
\text { Bronchogenic } \\
\text { carcinoma }\end{array}$} & & 46 true & 01 false positive & 47 \\
\hline & & positive & & \\
\hline & No & $\begin{array}{l}02 \text { false } \\
\text { negative }\end{array}$ & 04 true negative & 06 \\
\hline \multicolumn{2}{|l|}{ Total } & 48 & 05 & 53 \\
\hline
\end{tabular}

\begin{tabular}{|l|l|}
\hline INDICES \\
\hline Sensitivity & $95.83 \%$ \\
\hline Specificity & $80.00 \%$ \\
\hline Positive predictive value & $97.87 \%$ \\
\hline Negative predictive value & $66.67 \%$ \\
\hline
\end{tabular}

\section{DISCUSSION}

Bronchogenic carcinoma is the leading cause of cancer death in developed countries and is rising in alarming rates in developing countries. Patients with bronchogenic carcinoma have a poor prognosis wth an overall 5 year survival rate of 10 - $15 \%$. In our study attempt has been made to ascertain the CT characteristics, and histological typing of bronchogenic carcinoma.

CT evaluation of Tumor characteristics: In our study majority of the lesions were peripherally 
located $(73.5 \%)$ and the rest were found in a central location $(26.5 \%)$. This is in concordance with the study done by Vigg $\mathrm{A}^{12}$ where peripheral lesions are found to be more common than central lesions. Among peripherally located lesions squamous cell carcinoma was the most common in our study followed by adenocarcinoma. Usually adenocarcinoma is located peripherally.

About $64 \%$ of cases in our study had spiculated margins of the lesions in CT and 94\% of spiculated lesions were found to be Histopathologically positive for Bronchogenic carcinoma. Similar finding were seen in a study done by Shetty $\mathrm{CM}^{13}$. Cavitation is seen in about $18 \%$ of cases in our study and is seen most and is seen most common with squamous cell carcinoma. Calcification is identified in 4 cases out of the total 53 and among those 4 cases 2 were of Squamous cell carcinoma and other 2 were of benign pathology. Calcification in malignant cases could probably be dystrophic calcification. About $75 \%$ of cases showed moderate enhancement of the mass lesion in CECT and most of which were positive for malignancy by histopathology and $22 \%$ of cases showed only mild enhancement of the lesion

Pleural effusion was noted in about $23 \%$ of cases. Adrenals and bone were found to be the most common site of distal metastasis followed by liver in our study. About $88.7 \%$ of study subjects were diagnosed to have Bronchogenic carcinoma by $\mathrm{CT}$ and about $90.1 \%$ were diagnosed to have Bronchogenic carcinoma by Histopathology.

CT is a highly sensitive test (95.83\%) and also has a high positive predictive value $(97.87 \%)$ to diagnose Bronchogenic carcinoma from our study. But this increase in Positive predictive value (and a low Negative predictive value) may not be genuine and may be due to less number of Gold standard negatives in the sample studied which is a limitation of the study.

Histopathology and Staging : Method of specimen collection for Histopathology was by $\mathrm{CT}$ guided FNAC in 44 cases, Bronchoscopic biopsy in 8 cases and open Thoracotomy with wedge resection in 1 case. Pneumothorax was the most common complication associated with $\mathrm{CT}$ guided FNAC which was seen in 2 cases.

In our study squamous cell carcinoma is the most common histopathology followed by adenocarcinoma. This is in concordance with the study by Rawat et al ${ }^{14,15}$, Quamrul $\mathrm{M}^{16}$ and Shetty $\mathrm{CM}^{17}$

Table 30 Comparison of Various Histopathological Types of Bronchogenic Carcinoma between Present Study and Other Studies :

\begin{tabular}{|c|c|c|c|c|c|}
\hline & $\begin{array}{c}\text { Present } \\
\text { study }\end{array}$ & $\begin{array}{c}\text { Rawat et } \\
\text { al } 14\end{array}$ & $\begin{array}{l}\text { rishnamurt } \\
\text { hy } A^{51}\end{array}$ & $\begin{array}{c}\text { Quamrul } \\
\mathrm{M}^{16}\end{array}$ & $\begin{array}{l}\text { Shetty } \\
\mathrm{CM}^{13}\end{array}$ \\
\hline $\begin{array}{l}\text { Squamous cel } \\
\text { carcinoma }\end{array}$ & $45.3 \%$ & $44.8 \%$ & $15.6 \%$ & $66 \%$ & $44.4 \%$ \\
\hline $\begin{array}{l}\text { Adenocarcino } \\
\text { ma }\end{array}$ & $26.4 \%$ & $19.7 \%$ & $42.6 \%$ & $25.4 \%$ & $18.5 \%$ \\
\hline $\begin{array}{l}\text { Small cel } \\
\text { carcinoma }\end{array}$ & $16.9 \%$ & $16.75 \%$ & $13.2 \%$ & $3.4 \%$ & $17.2 \%$ \\
\hline $\begin{array}{ll}\text { Large } & \text { cel } \\
\text { carcinoma }\end{array}$ & $1.8 \%$ & $8.3 \%$ & $2.3 \%$ & $3.4 \%$ & $9.8 \%$ \\
\hline Others & $9.4 \%$ & $10.3 \%$ & $7 \%$ & $1.7 \%$ & 8.4 \\
\hline
\end{tabular}

Others include 5 cases which are Histopathologically negative for Bronchogenic carcinoma and it includes cases in which Histopatholgy reports came as inflammatory infiltrates without evidence of malignancy.Out of the 47 cases diagnosed as Bronchogenic carcinoma and staged by CT, 21 cases ( $44.7 \%$ ) were found to be of Stage IV which has poor prognosis

\section{CONCLUSION}

CT guided FNAC is a simple, less invasive and effective method to obtain Histopathology thereby prompting appropriate management.

CT is a highly sensitive test $(95.83 \%)$ and also has a high positive predictive value $(97.87 \%)$ to diagnose Bronchogenic carcinoma in a sample suitably selected and referred for malignancy.

Diagnostic work up by CT with guided FNAC may help the patient in correct diagnosis and thereby prompting appropriate management.

\section{BIBLIOGRAPHY}

1. Indian J Chest Disease Allied Sci 2004; 46 : 191-203. 
2. Travis WD,Brambilla E,Noguchi M,et al. International Association for the Study of Lung Cancer/American Thoracic Society/ European Respiratory Society: international multidisciplinary classification of lung adenocarcinoma executive summary. Proc Am Thor Soc 2011;8(5):381-5.

3. Michelle SG,Ravinder KG, Robert TH. Lung Cancer, Radiol Clin N.Am 45 (2007) 21- 43

4. Melissa L, Rosado C,Philip AT, Cesar A. Bronchogenic Carcinoma : Radiologic Pathologic Correlation, RadioGraphics $1994 ; 14$ : 429 - 446.

5. Edward F Patz. Imaging Bronchogenic Carcinoma. Chest $2000 ; 117$ (4_suppl 1); 90-95

6. William E B,Clyde A. Helms Fundamentals of Diagnostic Radiology, Third Edition; Lippincott Williams \& Wilkins, 2006, p444.

7. Mahoney MC, Shipley RT, Corcoran HL, Dickson BA.CT Demonstration Of Calcification In Carcinoma of The Lung,AJR Am J Roentgenol Feb 154 (2) : 255-8.

8. Khan AN, Al-Jahdali HH, Allen CM, Irion KL, Al Ghanem S, Koteyar SS. The calcified lung nodule: What does it mean?. Ann Thorac Med 2010;5:67-79.

9. Webb,Richard W.; Higgins, Charles B: Lung Cancer and Bronchopulmonary Neoplasms. Solitary and Multiple Nodules, Masses,Cavities, and Cysts,ebook Thoracic Imaging : Pulmonary and Cardiovascular Radiology. 1st ed,Philadelphia, Lippincott Williams \& Wilkins,2005 : 3,9.

10. Simon P.G. Padley,Olga Lazoura : Pulmonary Neoplasms : Grainger \& Allison`s Diagnostic Radiology,6th Edition, Churchill Livingstone Elsevier Limited,Vol 1, Chapter 15: 325-333.

11. Rivera MP, Mehta AC. Initial diagnosis of lung cancer: ACCP evidence- based clinical practice guidelines (2nd edition).
Chest 2007;132(3 Suppl):131S-48S.

12. Vigg A et al Pattern Of Lung Cancer In Elderly, J Assoc Physicians India October 2003 ; Vol 53 : 963 - 965.

13. Shetty C.M, Lakhkhar B.N, Gangadhar V.S.S, Ramachandran N.R. Changing pattern of bronchogenic Carcinoma : A Statistical Variation Or Reality. Indian J Radiol Imaging 2005 ;15 (1) : 233-238

14. Doll R, Hill AB. The mortality of doctors in relation to their smoking habits. Br Med J 1954;1(4877):1451

15. Rawat J, Sindhwani G, Gaur D, Dua R, Saini S. Clinicopathological Profile Of Lung Cancer In Uttarakhand. Lung India $2009 ; 26: 74-6$.

16. Jagdish R,Girish S,Dushyant G,Ruchi D,Sunil S. Clinico-pathological profile of lung cancer in Uttarakhand,Lung India. 2009 Jul-Sep; 26(3): 74-76.

17. Quamrul $M$ et al. Clinico-Pathological Profile Of Bronchogenic Carcinoma In a Tertiary Care Hospital In Bangladesh, Journal of Chittagong Medical College Teachers' Association $2010 ; 21$ (1) : 45 49 